\title{
L'accueil: regards croisés en France et au Brésil
}

\author{
Corinne Rougerie, (D) I, Roberta Carvalho Romagnoli, (D) II Cinira Magali Fortuna (DIII \\ ${ }^{I}$ Université de Cergy-Pontoise, Cergy-Pontoise, France

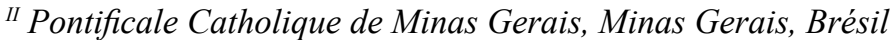 \\ III Université de São Paulo, São Paulo, Brésil
}

\begin{abstract}
Résumé
Cet article analyse l'accueil dans les services de santé et sociaux au Brésil et en France. L'accueil s'appuie sur des collectes d'informations qui amènent les personnes à livrer une part d'intimité de leur vie personnelle, de leur quotidien et de leur territoire. Il est souvent attribué à des agents peu ou surqualifiés mais des statuts d'agents administratifs ou d'animation de base. Dans les deux pays mis en regard, les équipes des secteurs de la Santé et du Social sont cloisonnées professionnellement entre service technique et service social. Les salariés sont alors divisés en fonction de leurs compétences professionnelles et de leur expertise. Les travailleurs ont des responsabilités différentes et la division n'est pas seulement liée à l'activité de travail, elle revêt aussi un caractère social reproduisant le clivage entre les classes sociales et leur hiérarchie professionnelle, dans une activité de travail peu valorisée.
\end{abstract}

Mot-clés: accueil; santé; service social; clivage socio professionnel; division du travail.

\section{O acolhimento: leituras cruzadas entre a França e o Brasil}

\begin{abstract}
Resumo
Este artigo analisa o acolhimento em serviços de saúde e social no Brasil e na França. O acolhimento se apoia em coletas de informações que leva as pessoas a revelarem aspectos íntimos sobre a vida da família, sobre seu cotidiano e sobre os territórios nos quais estão inseridas. Na França esse procedimento é frequentemente atribuido aos agentes do trabalho com menor qualificação profissional, mas com status de agentes administrativos ou formados em animação. Nos dois países analisados, as equipes do setor de saúde e do setor social estão "presas" entre dimensões técnicas e dimensões sociais. Os trabalhadores são divididos em função de suas competências profissionais e também de suas especialidades. Esses trabalhadores têm responsabilidades diferentes e a divisão não é somente ligada ao trabalho, mas é revestida também de um caráter social, reproduzindo a clivagem entre as classes sociais e suas hierarquias no cotidiano de trabalho, em uma atividade pouco valorizada.
\end{abstract}

Palavras-chave: acolhimento; saúde; serviço social; clivagem socioprofissional; divisão no trabalho.

\section{Embracement: Cross readings between France and Brazil}

\begin{abstract}
This article analyzes the user embracement in health and social services in Brazil and France. User embracement is based on information gathering that brings people to share intimacy with family life, their daily lives and the territory in which they live. It is often awarded to low or overqualified agents but on the status of basic administrative or animation agents. In both countries, the teams teams from the health and social sectors are professionally separated between technical and social services. Employees are then divided according to their professional skills and expertise. The workers have different responsibilities and the division is not only related to the activity of work, it also has a social character reproducing the cleavage between the social classes and their hierarchies in everyday work, in an activity that is not very valued.
\end{abstract}

Keyswords: user embracement; health; service social; socio-professional cleavage; division at work.

\section{Introduction}

En France, le service à la personne s'est institué progressivement comme un droit et un devoir du service public d'Etat. Les modalités d'accompagnement du public débutent par la rencontre du sujet avec l'institution sociale ou de santé. L'entrée en relation est la première modalité de la rencontre et se formalise par l'accueil dit de première ligne. Ces modalités d'accompagnement évoluent aujourd'hui avec les nouveaux modes de pilotage des institutions publiques et notamment la rationalisation budgétaire. Elles exigent des professionnels de s'inscrire dans une culture de résultat tout en maintenant une relation dite de qualité (GIULIANI, 2013). Les secteurs du champ social et de la santé sont traversés par des modalités de travail transposées du domaine

\footnotetext{
$\star$ Adresse de correpondance: Sinon Polaris formation 2rue du buisson 87170 Isl, France. E-mails: corirougerie@gmail.com, robertaroma@uol.com.br, fortuna@eerp.usp.b

Les données complètes des auteures sont disponibles à la fin de l'article.
}

de l'entreprise économique. Ils s'attachent à repenser la territorialisation de leurs organisations sous couvert des mêmes "bonnes" intentions, à savoir la garantie d'un service de proximité adapté aux besoins de l'usager. Des services d'état, aux collectivités territoriales, des établissements publics aux associations du secteur privé, chacun s'attèle à se restructurer, se regrouper, fusionner. Les pratiques dites de new management s'accompagnent d'un repositionnement du sujet que ce dernier subit paradoxalement le plus souvent. D'usager à destinataire d'une offre de service, la personne se retrouve malgré elle au cœur de la division du travail, chaque situation étant considérée comme normalisable (WELLER, 2010).

$\mathrm{Au}$ Brésil, l'accueil devient central dans la vie quotidienne du service après les modifications dans la Constitution de 1988 et dans les politiques publiques suivantes. Comme soulignent Neves et Herckert (2010), traditionnellement cette pratique se présente comme une attitude 
volontariste, mis en place pour rendre service à des usagers. Elle repose souvent sur la bienveillance de certains professionnels. Au cours des dernières années, nous avons vu des débats autour de l'accueil qui est maintenant considéré comme une action importante pour résoudre les problèmes d'accès et réduire les listes d'attente pour ces services. La mission d'accueil participerait désormais à promouvoir le changement dans le processus de travail en essayant de répondre à tous ceux qui cherchent des services et une garantie de leurs droits sociaux. La plupart du temps, l'accueil est abordé dans sa dimension spatiale, comme une action de triage administratif et un transfert des demandes vers des services spécialisés.

La notion de guichet unique est donc un point commun aux deux pays. L'accent mis sur l'accueil confond souvent cette pratique avec l'accès qui implique divers aspects (géographique, économique, socio-culturelle, fonctionnelle). Par exemple, la garantie de l'accès aux soins est souvent limitée aux lignes générales des textes officiels. L'accueil dans le quotidien des services sous-entend pourtant une réorientation individualisée des actions en faveur de la personne, une pratique de responsabilité de toute l'équipe des professionnels et la promotion de l'intégration des connaissances et des pratiques.

Nous nous proposons de discuter les tensions institutionnelles que fait émerger l'activité d'accueil. Les différences et les similitudes entre les deux pays nous permettent de mettre en perspective les pratiques d'accueil et interrogent plus globalement la professionnalité de personnels du champ social et de la santé. Nous postulons que les pratiques d'accueil de ces deux champs sont intrinsèquement et historiquement reliées par la notion du "care". Le "care" regroupe les situations qui requièrent un investissement relationnel étalé dans la durée et s'oppose au curatif "Cure" un modèle centré sur le soin (ROTHIER BAUTZER, 2012).

Nous apporterons dans un premier temps des éléments de contextualisation afin de saisir les problématiques étatiques de ces deux pays. Nous poursuivrons par la définition et l'analyse de l'activité d'accueil. Nous nous attacherons enfin à comprendre comment cette activité impacte la professionnalité des salariés chargés de la mettre en place tant au niveau du champ social que de la santé et au regard du contexte brésilien et français.

\section{Eléments de contextualisation: l'accueil en France et au Brésil dans le champ du travail social et de la santé}

\subsection{Le contexte social et de santé français}

En France, les départements (conseils départementaux) sont devenus progressivement pilotes de l'action sociale. La deuxième vague de décentralisation de 2003 a amené un redécoupage des compétences, accentuant leur domination dans ce domaine. Le système de financement repose sur un système assuranciel et d'assistance. Il conduit à une division, voire à une catégorisation des personnes insérées ou inadaptées en fonction de leur rapport au travail. Par convention, des missions de l'action sociale sont déléguées aux communes via leur Centre Communal d'Action Sociale (ROUGERIE, 2015). La dimension territoriale et de proximité de ce type d'établissement public permet d'adapter localement des politiques sociales aux besoins des populations. Le CCAS est aussi l'un des premiers établissements en France à développer historiquement un accueil dit de proximité. Il dépend de la fonction publique territoriale et anime à ce titre une action générale de prévention et de développement social sur le territoire communal ou intercommunal, en liaison avec les institutions publiques et privées. Selon l'article 123-4 du code de l'action sociale et des familles, il développe différentes activités et missions légales ou facultatives, directement orientées vers les populations concernées.

Dans ce paysage, les questions de santé demeurent pourtant l'apanage du domaine dit de santé publique d'Etat renforcé par le cadre législatif de la fin des années 2000 au risque d'une "dilution du médicosocial". Le risque est de soumettre à terme le secteur social à l'instauration de modes opérationnels concrets et évaluables du secteur médical (BARREYRE, 2014). La loi Hôpital, Patient, Sanitaire et Territoires dite HPST de 2009 en est l'illustration en imposant la coopération entre les secteurs sanitaire et social, sans moyens supplémentaires. Toutefois, elle ne pallie pas aux inégalités de couverture médicale sur les territoires. Certains secteurs ruraux restent encore mal desservis en services de santé et de proximité. L'emprise historique, culturelle et récurrente des métiers du sanitaire sur ceux du social freine les collaborations rendues nécessaires par la paupérisation des situations, leur complexité et leurs impacts sur la santé des personnes.

L'une des conséquences directes est la généralisation de guichet unique afin de répondre efficacement aux besoins globaux des personnes. Le principal effet dans les services publics dits d'Etat est d'envisager l'accueil comme une activité professionnelle soumise à évaluation, comme prestation de service. Depuis 2005, ces services peuvent solliciter un label qualité à travers une charte qui prend la forme d'un référentiel en 2013 soumis à cinq principes: horaires adaptés, accueil courtois, informations claires, délais garantis et écoute permanente. A ce titre, l'accueil est considéré comme la première étape de toute prestation de service au public (FRANCE, 2013). Il interroge autant le volet qualitatif de l'entrée en relation que les modalités de sa mise en œuvre en termes de compétence et qualification par les agents chargés de sa mise en œuvre. Il porterait des injonctions: travailler en partenariat et en réseau, décloisonner les pratiques tout en imposant un développement accru de l'évaluation et de l'auto-évaluation, du travail de proximité, de la mobilisation des usagers. Depuis fin 2016, un plan en faveur du travail social et du développement social entérinerait cette tendance, en la généralisant à l'ensemble des services publics à caractère social. Un "premier accueil social" est plébiscité en insistant sur son aspect inconditionnel, ancré obligatoirement sur le territoire et en proposant l'instauration une charte de qualité (FRANCE, 2016).

Fractal, Rev. Psicol., v. 31 - n. 2, p. 53-59, 2019 


\subsection{Le contexte Brésilien}

Au Brésil, nous avons une Constitution citoyenne de 1988 issue d'une vaste mobilisation sociale qui a permis d'instituer un système de sécurité sociale (BRASIL, 1988). Pour la première fois, la santé est reconnue comme un droit et l'assistance sociale comme universelle.

Pour la santé, le Système Unique de Santé (SUS) a été mis en œuvre, composé par les trois niveaux de gouvernement, municipal, étatique et fédéral, et le secteur privé contracté et conventionné, exercent conjointement une action selon les principes d'universalisation, d'équité, d'intégralité, de décentralisation et de participation populaire.

Depuis 1990, beaucoup d'efforts ont été réalisés pour construire un réseau de santé avec des services de premier recours (les équipes de santé de la famille, des centres de santé, unités basique de santé, etc.), des services spécialisés (ambulatoires), des services d'urgence et les hôpitaux.

Le pays est très diffèrent en réalité en termes de ressources et de disponibilités des professionnels de la santé. Les régions plus pauvres ont des difficultés pour mettre en œuvre les actions de santé de prévention, promotion et care, c'est le cas du nord et nord-est du pays.

Un autre défi est celui de la politique néolibérale mise en place dans les dernières années pour les gouvernements et concerne la mise en ouvre de la logique de la nouvelle gestion publique dans le secteur de la santé.

Le financement du système unique de santé n'a jamais été prévu pour faire face aux besoins de santé du peuple brésilien.

La santé de la famille et les autres stratégies pour modifier le modèle de santé centré sur la maladie et sur les aspects biologiques ont été mis en œuvre. L'accueil a été instauré comme dispositif à travers la politique nationale d'humanisation de la santé.

De l'autre côté, l'assistance sociale connait un grand changement après les années 1990 suite à la configuration de la protection sociale égale et universelle, soutenue dans la Constitution de 1988 Jusqu'alors, le système était caractérisé par le clientélisme particulier, par l'absence de paramètres universalistes et de transparence dans les actions et le manque de participation de la société civile.

Ce modèle a eu des conséquences néfastes pour la société brésilienne, puisqu'il s'est révélé inefficace dans la lutte contre l'exclusion sociale et dans la garantie des droits minimaux des citoyens. Le changement de cette conception a fait "[...] la transformation de la charité, des bienveillances et de l'aide à la notion de droits et de citoyenneté dans le domaine de l'assistance sociale en soutenant le caractère de politique publique de la protection sociale articulée à d'autres politiques visant à garantir les droits et conditions de vie decentes" (CRUZ; GUARESCHI, 2009, p. 27).

Dans ce contexte, après une forte mobilisation populaire dans les années quatre-vingt-dix, l'assistance sociale est instituée en tant que politique publique et la protection sociale devient un droit pour tous, sans contribution préalable. La politique publique de l'assistance sociale s'organise selon trois axes: les personnes, leurs besoins et la famille, dans le but de garantir les droits et de promouvoir la citoyenneté auprès de segments exclus de la société. Le Système Unique d'Assistance Sociale (SUAS) a été implanté en 2005 et comprend des actions de vigilance sociale qui visent à prévenir des situations de risque social, par le biais des potentialités et du renforcement des liens affectifs et familiaux, et des actions d'accueil socio-assistancielles destinées à des individus et des familles en situation de violation de droits (BRASIL, 2004). Parmi les formes de protection sociale que le SUAS fournit, il y a la Protection Sociale Basique (PSB) et l'équipement pour sa mise en pratique est le Centre de Référence en Assistance Sociale (CRAS). Le CRAS est une référence pour le développement de tous les services socio-assistanciels à caractère préventif, protecteur et proactif, en intervenant sur les potentialités des sujets, des familles et des communautés. Ce centre est considéré comme la porte d'entrée du domaine de l'Assistance Sociale et il est implanté dans la commune en zone de vulnérabilité sociale. Le CRAS se destine à des segments de la population qui vivent dans des conditions telles que la pauvreté, la privation (absence de revenus, accès nul ou précaire aux services publics) et la fragilisation des liens affectifs (discrimination d'âge, ethnique, de genre ou par déficience). Son intervention est organisée en deux dimensions articulées et associées avec le territoire: la dimension socio-assistancielle (soutien aux usagers et potentialisation de réseau des services pour assurer l'accès aux droits) et la dimension socio-éducatif (approche des usagers en tant que sujet socio-culturel, en visant leur inclusion dans services de réseau et de l'inclusion sociale).

En effet, dans le CRAS, l'équipement qui a pour mission la réception des familles vulnérables, l'accueil y prend une place importante. Il est défini dans les documents officiels comme "processus d'écoute initiale des besoins et des demandes introduites par les familles, tout en offrant des informations sur les actions du Service, le réseau d'assistance sociale, en particulier les autres politiques sectorielles CRAS" (BRASIL, 2012, p. 17). L'accueil apparait donc identifié dans des cadres institués.

\subsection{Un enjeu commun: l'accueil un analyseur des besoins sociaux et de santé sur le territoire}

L'analyseur est compris au sens entendu en Analyse Institutionnelle. Il est un élément ou des situations qui se présentent et peuvent révéler des contradictions et les relations de pouvoir existantes dans les institutions (SPAGNOL et al., 2016). Au Brésil comme en France l'accueil prétend comprendre les multiples demandes présentées par les familles, l'identification des rapports entre les vulnérabilités; les ressources et les capacités sur le territoire. Il nécessite le recueil d'informations par des entretiens plus ou moins prolongés, des évaluations socio-économiques et des propositions d'orientations. La procédure d'accueil devient informatisée (dossier en ligne, etc.). L'accueil s'appuie donc sur des collectes d'informations qui amènent les personnes à livrer une part d'intimité sur la vie de famille et sur le territoire. Parmi les conditions 
factuelles de l'accueil, les textes et règlement intérieurs insistent sur les dimensions spatiales (salle agréable et conviviale) mais aussi sur le respect des règles de confidentialité. Une exigence particulière est demandée en matière d'écoute, d'empathie, d'expertise et d'évaluation et d'orientation. Ces compétences exigées sont clairement identifiées, par exemple, dans les fiches de postes consultées dans une recherche sur l'accueil, menée en CCAS, auprès des agents les moins qualifiés (ROUGERIE, 2015). L'activité d'accueil revêt une fonction d'observatoire social sur les relations de la famille avec le territoire, la compréhension de la réalité vécue entre les familles et les institutions.

$\mathrm{Au}$ Brésil, le développement des actions communautaires impose un accueil en groupe pour partager des problèmes. Par exemple, les familles sont invitées à partager et identifier des exigences communes. L'objectif est double : ne pas individualiser les problèmes et favoriser leur autonomie et leur participation sociale à la réalisation de leurs droits. Dans ce contexte, l'accueil montre une fonction prépondérante, celle du soutien psychosocial. Il contribue à l'émergence et au développement d'un processus d'accompagnement (orientation, guides, l'inscription dans les programmes sociaux, les visites...). L'accueil est le maillon entre l'institution et l'usager. Ce lien est essentiel pour sortir de la logique d'assistance et ou de tutelle. Il participe à la construction de l'autonomie pour affronter la vulnérabilité sociale. Bien que l'accueil soit prévu par le cadre législatif, il existe un fossé entre le cadre institué et sa mise en œuvre réelle par les équipes pluriprofessionnelles.

Au Brésil, comme en France, l'accueil est modifié par les réalités socio-économiques du territoire. Ce dernier est plus qu'un espace géographique. Il est composé par les relations sociales entre les personnes. Le territoire correspond à des espaces de vie, de relations, d'échanges, de construction et de déconstruction des liens quotidiens, de conflits et de contradictions qui révèlent les significations attribuées par différents sujets. Dans un territoire, on retrouve la complexité de la façon de vivre, de travailler, de soigner, les coutumes, les références pour être et vivre ensemble. Dans ce contexte, il est toujours nécessaire que les professionnels appréhendent le territoire. Mais ils ont souvent des difficultés à connaître et à se connecter à cette dimension qui impose de savoir et pouvoir créer des liens singuliers entre eux et les usagers. Les difficultés sont associées au fait que le territoire est en mouvement et en changement. Bien que la taille du territoire diffère d'un pays à l'autre, les soignants comme les travailleurs sociaux doivent être associés à l'analyse des besoins de santé et sociaux en considérant leur propre spécificité. Par exemple, les conditions de santé ou de vulnérabilité des familles ne font pas référence à une logique relationnelle statique. Bien que les documents suggèrent aussi une lecture subjective de la famille, les professionnels insistent sur les perceptions du territoire qui sont essentielles pour créer des liens entre les usagers et le service et aussi la réalisation d'actions collectives (ABADE, 2016).
Ce détour par le cadre institutionnel nous permet de montrer les liens entre la construction de la relation de proximité avec le public dans un contexte étatique, de type "poupée gigogne" ou de millefeuille dans lequel l'usager doit trouver son chemin. Il montre aussi l'évolution progressive du territoire et sa transformation qui semble poser les mêmes enjeux en matière de politique d'accueil en France et au Brésil, dans le champ de l'action sociale et de la santé. Il interroge les pratiques d'accueil et leurs modalités non plus centrées uniquement sur l'individu mais un groupe d'individus.

\section{Du pré accueil à l'accueil: une activité professionnelle difficilement identifiable}

\subsection{Les modalités d'accueil: ou comment prendre en compte la vulnérabilité des sujets}

L'accueil demeure confiné à une relation duelle, dans le contexte français. Il a évolué vers deux fonctions. La première est administrative. Elle correspond au modèle "d'auxiliaire social" (remplissant des tâches de bilan d'orientation, d'instruction de dossiers, d'engagement de procédures). La seconde relève de l'animation par la mise en relation des acteurs, diffusion des informations, détection des demandes, médiation sociale (MAUREL, 2000). Le premier modèle est fréquemment développé dans les administrations publiques. Il est le plus souvent réalisé au guichet. Ces deux fonctions peuvent se chevaucher comme nous avons pu l'observer lors d'une recherche menée en CCAS (ROUGERIE, 2015). Il est souvent attribué à des agents peu ou surqualifiés mais sur des statuts d'agents administratifs ou d'animation de base. Ces professionnels réalisent un accueil dit en front office, un premier accueil (CONSEIL SUPERIEUR DU TRAVAIL SOCIAL [CSTS], 2007). Les travailleurs sociaux sont en back office qui prend souvent la forme de permanence sociale sur rendez-vous (RULLAC; OTT, 2015). Ces types d'accueil sont le plus souvent soumis à la responsabilité et à la liberté de l'usager, dans un contexte de pressurisation face aux demandes exponentielles dans certains quartiers. Les permanences en travail social, dites en polyvalence de secteur, développent des systèmes de pré-accueil pour éviter de passer à côté de demandes d'urgence sociale (CSTS, 2007). Les modalités d'accueil s'apparentent à des dispositifs, à savoir des espaces de travail organisés en fonction de missions pré-établies, une modalité d'action publique mais qui n'a pas de caractère imposé. En France par exemple, les permanences sans rendez-vous des travailleurs sociaux de la Fonction Publique se raréfient. Ce sont les espaces dits de "pré accueil" qui reçoivent les premières demandes et doivent traiter, analyser les données et orienter, souvent avec une impression d'urgence, et dans l'instantanéité (ION; LAVAL; RAVON, 2007). Ce n'est pas tant l'urgence d'intervenir qui prime, mais la "reconnaissance" nécessaire de la personne dans toute sa vulnérabilité conduisant aux paradoxes de prises en charge déjà relevés. La vulnérabilité est comprise alors comme un concept opératoire "analyseur des problèmes sociaux contemporains" pouvant se définir sous sa forme essen- 
tialiste comme: le fruit d'un rapport situé et daté entre des propriétés individuelles et les propriétés du contexte. En d'autres termes, cela revient à dire que des individus singuliers sont vulnérables dans certaines conditions et dans celles-ci seulement (SOULET, 2014).

Dans les faits observés l'accueil reste parfois soumis à des dimensions qui relèvent du "cure" comme nous pouvons le relever à partir du contexte français. Le travail du "care" et du "cure" sont des modèles empruntés historiquement au milieu médical. L'histoire du service social nous rappelle les liens indissolubles entre travail social et santé. Les métiers et les qualifications professionnelles se sont d'abord structurés des infirmières visiteuses aux assistantes sociales. L'entrée en relation avec les usagers s'est construite à partir d'une logique d'intervention ciblée sur l'assistanat et le secours d'urgence.

Au Brésil, l'accueil dans le CRAS est perçu comme très important pour les équipes qui pensent que les conditions actuelles des actions d'accueil sont meilleures que celles ayant traditionnellement existé dans l'assistance sociale (CRUZ; SCHIEFFERDECKER, 2011). Une des difficultés est due à la formation encore très attachée à une vision biologique de la santé et maladie et réduite $\mathrm{du}$ social. Les problèmes sociaux sont parfois compris comme une responsabilité individuelle de chaque citoyen. Ainsi la pauvreté est définie et attribuée en fonction des individus et non en lien avec les conditions générales de vie. Avoir une bonne santé relèverait d'un choix et non d'un droit: il faudrait simplement avoir des bonnes habitudes comme une nourriture saine, de l'exercice physique quotidien, du repos, des loisirs etc. Pour avoir du travail, il suffirait de savoir préparer son parcours professionnel, d'avoir de la patience pour chercher un emploi etc. Mais il faut souligner que la vulnérabilité sociale est un concept complexe qui englobe de multiples dimensions d'ordre économique, environnemental, de santé, de droits individuels et sociaux... Ces conditions amplifient les probabilités qu'il en découle d'autres et développent donc d'autres précarités. Plus une famille est vulnérable, plus elle peut s'exposer à des risques, à des situations qui peuvent atteindre le bien-être et la santé des individus, des familles, des groupes et des communautés. Être en vulnérabilité sociale revient à avoir, potentiellement, la réponse altérée ou diminuée face aux situations de risque ou de contraintes naturelles de la vie. La vulnérabilité sociale recouvre des situations multi déterminées composées d'éléments transversaux de plusieurs ordres qui mènent constamment à des heurts. Les professionnels sont alors amenés à développer des stratégies et des actions en SUAS et en SUS pour chercher à créer les conditions pour renforcer la capacité d'agir des usagers.

Les politiques publiques brésiliennes et françaises proposent l'accueil comme capacité d'intervenir selon le besoin de chaque personne, une façon de traiter les gens avec l'humanisation, mais dans la pratique il y a des contradictions et l'accueil devient un "entonnoir" aux ressources sociales et de santé. En d'autre termes, il participe à catégoriser les publics en excluant volontairement ou non une partie de la population qui ne bénéficie pas ou plus des services publics de droits. L'accueil ne se réduit pas à une tâche rationnelle, bureaucratisée. Il suggère une relation humaine. Si celle-ci est à visée transformative, elle induit un changement autant chez l'accueilli que chez l'accueillant.

\section{L'accueil, un métier transversal?}

En France et au Brésil l'ensemble de ces accompagnateurs sociaux et de santé partage les mêmes modalités matérielles et spatiales de face à face et les mêmes domaines d'intervention habituellement séparés de l'action sanitaire et sociale. Par contre, les statuts ne se différencient plus à l'intérieur d'un même champ. Un travailleur social initié et l'agent d'accueil administratif peuvent effectuer un accueil de première ligne. Ce sont dans ces interstices de la prise en charge que les métiers de "première ligne" ou "métiers du front" apparaissent (MAUREL, 2000). Des sociologues travaillant sur le champ de l'action sociale s'intéressent indirectement à l'accueil comme une fonction pour analyser le travail social ou la relation d'aide. Ravon emploie par exemple le terme de rencontre pour décrire la fonction d'accueil (RAVON ; RAYMOND, 1997). L'accueil est vécu comme une activité professionnelle empêchée par l'intensification des demandes engendrant à terme du mal-être voire de la violence au travail (WELLER, 2010). La formalisation des tâches et l'informatisation, la montée des demandes financières et sociales induisent de nouvelles modalités de travail qui influent sur l'entrée en relation. Les travailleurs sociaux français par exemple se retrouvent de plus en plus en situation de back office, laissant craindre une perte de leur identité professionnelle spécifique (CHOPART, 2000). L'analyse de l'activité d'accueil se centralise en France sous l'angle de l'organisation de travail et de ses conséquences sur les usagers et les agents d'accueil. Elle fait ressortir la pénibilité du travail, statistiques nationales à l'appui et corrobore l'articulation organisation de travail et mode de management. On est passé en France d'un temps d'accueil bureaucratisé et rationnel dans les années 1980 à celui du temps de la relation de service dans les années 1990. L'accueil de contact aujourd'hui s'inscrit dans une volonté d'individualisation et de personnalisation du traitement des situations tout en généralisant et vulgarisant une action d'aide dévolue jusqu'alors aux travailleurs sociaux dits de "cœur de métiers" (ROUGERIE, 2015). Dans ce contexte, une nouvelle disponibilité d'activité d'accueil s'offre pour les agents administratifs qu'ils soient personnels issus du champ social ou médical. Deux invariants résisteraient au temps: l'autonomisation et la professionnalisation du travail de la relation se développent dans une logique socio historique: "la relation fait lien social" et la rémunérer comme travail participe de l'intégration économique, gestionnaire, symbolique et morale (DEMAILLY, 2008). Dans le champ du travail social, l'activité d'accueil demeure pourtant difficilement identifiable en termes de statuts et de qualifications spécifiques. On peut la définir comme une fonction commune et partageable à toute institution en contact direct avec des personnes en demande d'aide (MAUREL, 2000). Cette approche nous parait 
pouvoir être généralisée aux deux pays. Ainsi dans les deux pays mis en regard ici, les équipes des deux secteurs (Santé et Social) sont composées par une division technique et sociale du travail avec des professionnels divisés en fonction de leurs compétences professionnelles, de leur expertise. Les travailleurs ont des responsabilités différentes et la division n'est pas seulement technique du travail, il y a aussi une division sociale qui reproduit les classes sociales dans le travail (MATOS; PIRES, 2006). Mais les collectifs de travail restent globalement actifs contrairement à ce que l'on peut observer ordinairement dans les études sur la souffrance au travail. Ils concourent à mettre en opposition travailleurs sociaux et agents administratifs, infirmiers et auxiliaires de soins par exemple dans une optique concurrentielle, celle de la lutte des places (GAULEJAC; BLONDEL; TABOADA-LEONETTI, 2014).

\section{Considérations finales}

Comme nous avons vu, l'accueil dans les différents pays est un processus singulier, impliquant la confrontation de contradictions et des tensions. Nous sommes face à une situation complexe où il n'est pas facile d'intervenir selon le besoin des usagers, avec humanisation et en tenant compte de la spécificité des territoires. Soutenir les champs de forces en présence dans l'accueil est essentiel. Nous pensons que cette posture est importante afin d'échapper à la logique de la tutelle de l'Etat et de soutenir des approches professionnalisant davantage l'accueil, participant par la même d'un travail d'émancipation et d'appropriation par le sujet de son propre pouvoir. L'accueil demande à être repensé comme un métier à part entière à l'interface du social et de la santé. Plus les personnes sont reçues et orientées au plus près de leurs besoins et mieux elles peuvent conjuguer avec les services publics. En réduisant les intermédiaires professionnels par un accueil professionnalisé géré par des agents qualifiés et/ou requalifiés, nous pensons que les parcours sociaux et de santé s'apparenteront de moins en moins à des impasses administratives, juridiques et psychologiques dans lesquelles les personnes sont conduites malgré elles. Ces perspectives amènent à repenser l'accueil dans les cursus de formation des professionnels de la santé et du social, en favorisant et en mutualisant les passerelles entre les deux univers professionnels, toujours très cloisonnés.

\section{Informations sur les auteurs:}

Corinne Rougerie

(iD https://orcid.org/0000-0001-5253-6454

$\mathrm{R}^{\mathrm{G}}$ https://www.researchgate.net/profile/Corinne_Rougerie

Chercheure associée, Laboratoire EMA (EA. 4507 Education Mutation Apprentissages) Université de Cergy Pontoise, France.

Roberta Carvalho Romagnoli

(iD) https://orcid.org/0000-0003-3551-2535

(9) http://lattes.cnpq.br/0924610511932717

Professeur au Département de Psychologie à l'Université Pontificale Catholique de Minas Gerais (PUC Minas), Brésil

\section{Cinira Magali Fortuna \\ (iD) https://orcid.org/0000-0003-2808-6806 \\ (9) http://lattes.cnpq.br/2878561750710139}

Professeur au Département Maternelle, Infantile et de Santé Publique de 1'Ecole Sciences Infirmières de Ribeirão Preto de L'Université de São Paulo (USP), Brésil

\section{Contribution des auteurs:}

Tous les auteures ont collaboré tout au long du processus, de l'élaboration à la révision finale du manuscrit. Les auteurs ont approuvé le manuscrit final pour publication.

\section{Comment citer cet article:}

\section{ABNT}

ROUGERIE, Corinne; ROMAGNOLI, Roberta Carvalho; FORTUNA, Cinira Magali. L'accueil: regards croisés en France et au Brésil. Fractal: Revista de Psicologia, Niterói, v. 31, n. 2, p. 5359, maio/ago. 2019. https://doi.org/10.22409/1984-0292/v31i2/28577 APA

Rougerie, C., Romagnoli, R. C., \& Fortuna, C. M. (2019). L'accueil: regards croisés en France et au Brésil. Fractal: Revista de Psicologia, 31(2), 53-59. doi:10.22409/1984-0292/v31i2/28577

\section{Références}

ABADE, Flávia Lemos. Os sentidos das ações coletivas do PAIF. 2016. Tese (Doutorado em Psicologia)-Pontifícia Universidade Católica de Minas Gerais, Belo Horizonte, Minas Gerais, 2016.

BARREYRE, Jean-Yves. Eloge de l'insuffisance : Les configurations sociales de la vulnérabilité. Paris: Eres, 2014.

BRASIL. Constituição da República Federativa do Brasil. Brasília: Senado Federal, 1988.

BRASIL. Ministério do Desenvolvimento Social e Combate à Fome. Política Nacional de Assistência Social. Brasília: Secretaria Nacional de Assistência Social, 2004.

BRASIL, Ministério do Desenvolvimento Social e Combate à Fome. Orientações Técnicas sobre o PAIF. Brasília: Secretaria Nacional de Assistência Social, 2012.

CHOPART, Jean-Noël (Dir.). Introduction. In: Les mutations du travail social : dynamiques d'un champ professionnel. Paris: Dunod, 2000. p. 1-8.

CRUZ, Lílian Rodrigues da; GUARESCHI, Neuza Maria de Fátima (Org.). A constituição da assistência social como políticas públicas: interrogações à Psicologia. In:

Políticas públicas e assistência social. Petrópolis, RJ: Vozes. 2009. p. 13-40.

CRUZ, Lilian Rodrigues da; SCHIEFFERDECKER, Márcio André. Práticas psicológicas e políticas públicas de assistência social: algumas interrogações. In: JORNADA DE PESQUISA EM PSICOLOGIA: DESAFIOS NAS PRÁTICAS DA PSICOLOGIA, 4, 2011, Universidade de Santa Cruz do Sul. Anais... Rio Grande do Sul, 2011. p. 137-142.

CONSEIL SUPERIEUR DU TRAVAIL SOCIAL (Dir.). Ministere du Travail, des Relations Sociales, de la Famille, de la Solidarite et de la Ville. Le travail social confronté aux nouveaux visages de la pauvreté et de l'exclusion. Rennes: Presses de l'EHESP, 2007. 
DEMAILLY, Lise. Politiques de la relation: approche sociologique des métiers et activités professionnelles relationnelles. Paris: Septentrion, 2008.

FRANCE. Secrétariat Général pour la Modernisation de l'Action Publique. Référentiel Marianne. v. 3, juin 2013. Disponible en: https:/ www.modernisation.gouv.fr/sites/default/files/fichiers-attaches/ referentiel_marianne_vf_juin_2013.pdf. Accès le 1 juil. 2016.

FRANCE. Ministere des Affaires Sociales et de la Santé. Secrétariat d'État chargé des Personnes handicapées et de la Lutte contre l'Exclusion. Guide d'appui à la structuration du premier accueil social inconditionnel de proximité. 2016. Disponible en: https://solidarites-sante.gouv.fr/IMG/pdf/ guide_accueil_social_proximite.pdf. Accès le 13 août. 2017.

GAULEJAC, Vincent de; BLONDEL, Frédéric; TABOADALEONETTI, Isabel. La lutte des places. Paris: Desclée De Brouwer, 2014

GIULIANI, Frédérique. Accompagner : le travail social face à la précarité durable. Paris: Presses Universitaires de Rennes (Le sens social), 2013.

ION, Jacques; LAVAL, Christian; RAVON, Bertrand. Politiques de l'individu et psychologies d'intervention: transformation des cadres d'action dans le travail social. In : CANTELLI, Fabrizio ; GENARD, Jean-Louis. (Coord.). Action publique et subjectivité. Paris: Éd. Droits et Société, 2007. p. 157-168.

MATOS, Eliane; PIRES, Denise. Teorias administrativas e organização do trabalho: de Taylor aos dias atuais, influências no setor saúde e na enfermagem. Texto \& Contexto - Enfermagem, Florianópolis, v. 15, n. 3, p. 508-514, set. 2006. http://dx.doi. org/10.1590/S0104-07072006000300017.

MAUREL, Elisabeth. Les métiers de l'accueil. In: CHOPART, Jean-Noël (Dir.). Les mutations du travail social: dynamiques d'un champ professionnel. Paris: Dunod, 2000. p. 125-138.

NEVES, Claudia Abbes Baeta; HECKERT, Ana Lúcia Coelho. Micropolítica do processo de acolhimento em saúde. Estudos e Pesquisas em Psicologia, Rio de Janeiro, v. 10, n. 1, 2010. Disponible en: http://pepsic.bvsalud.org/scielo.php?script=sci arttext\&pid=S1808-42812010000100011\&lng=pt\&nrm=iso. Accès le 27 juil. 2017.

RAVON, Bertrand ; RAYMOND, Roland. Engagement bénévole et expérience de soi. L'exemple des Restaurants du cœur. In: ION, Jacques. PERONI, Michel. (Dir.). Engagement public et exposition de la personne. La Tour d'Aigues: Éd. de l'Aube, 1997. p. 99-109

ROTHIER BAUTZER, Éliane. Entre cure et care, les enjeux de la professionnalisation infirmière. Paris: Université Paris Descartes, 2012.

RULLAC, Stéphane; OTT, Laurent. Dictionnaire pratique $d u$ travail social. Paris: Dunod, 2015.

SPAGNOL, Carla Aparecida et al. Dispositif socianalytique: instrument d'intervention et de collecte de données en recherche qualitative en soins infirmiers. Recherche en soins infirmiers, v. 124, n. 1, p. 108-117, 2016. https://doi.org/10.3917/rsi.124.0108

ROUGERIE, Corinne. L'accueil: un analyseur des implications professionnelles dans le travail social. Recherche socioclinique en CCAS (Centre Communal d'Action Sociale). 2015. $363 \mathrm{f}$. Thèse (Doctorat en Sciences de l'Education)-Laboratoire EMA, Université de Cergy-Pontoise, Cergy-Pontoise, 2015. Disponible en : https://www.theses.fr/2015CERG0833.pdf. Accès le 24 avril 2017.

Fractal, Rev. Psicol., v. 31 - n. 2, p. 53-59, 2019
SOULET, Marc-Henry. Les raisons d'un succès. La vulnérabilité comme analyseur des problèmes sociaux contemporains. In: BRODIEZ-DOLINO, Axelle et al. (Dir.). Vulnérabilités sanitaires et sociales: de l'histoire à la sociologie. Rennes: PUR, 2014. p. 59-64.

WELLER, Jean-Marc. Comment les agents se soucient-ils des usager?. Informations sociales, v. 158, n. 2, p. 12-18, 2010. Disponible en: https://www.cairn.info/revue-informationssociales-2010-2-page-12.htm. Accès le 28 avril 2017. 